\title{
Rectenna Design for Enhanced Node Lifetime in Energy Harvesting WSNs
}

\author{
Prakash K Sonwalkar ${ }^{1}$, Vijay Kalmani ${ }^{2}$ \\ Research Scholar, VTU Belagavi ${ }^{1}$, \\ Department of Computer Science and Engineering \\ Jain College of Engineering, Belagavi - India ${ }^{1,2}$
}

\begin{abstract}
In a scenario where every possible solution is investigated for sustainability, Energy Harvesting (EH) stands as an undisputed candidate for enhancing the network lifetime in WSNs where node lifetime decides the network's life. Radio Frequency (RF) energy is abundantly available in the ambience among all the available energy sources. Since both information and power are transmitted in an RF signal, EH is possible in the far-field region. At first, we present a novel 4-element rectangular Patch Antenna Array (PAA) design of EH rectenna. The receiving antenna is designed to pick up the radio signal in the RF range (2.45 GHz) from the free space. Then, the H-shape antenna is modified by introducing a circular slot to enhance the bandwidth. The paper compares the results of the basic parameters of the antenna, such as return loss, input impedance, bandwidth, gain, directivity, and efficiency. As a result, the modified H-shaped antenna (with circular slot) has an increased gain from $8.24 \mathrm{~dB}$ to $8.32 \mathrm{~dB}$, with a reduced return loss from $-10 \mathrm{~dB}$ to $-16 \mathrm{~dB}$ and enhanced bandwidth from $64.8 \mathrm{MHz}$ to $868 \mathrm{MHz}$. The high gain, large bandwidth, suitably matched impedance for a minor return loss, and high efficiency of the modified $\mathrm{H}$-shaped patch antenna makes it eligible for energy harvesting application.
\end{abstract}

Keywords-Antenna design; backscattering; beamfroming; energy harvesting; sequential rule; wireless sensor networks

\section{INTRODUCTION}

With the proliferation of edge devices and extensive study on deployment, WSNs find their applications ranging from remote applications to body area networks. A typical WSN intends to monitor the environment with the aid of sensor(s), micro-controller(s), transceiver data storage, and energy storage facilities (batteries). The battery acts as an energy source for a node, and its power decides the life of a WSN. Therefore, energy harvesting is perceived as an amicable solution for the bottleneck created by the limited lifetime of the battery. Recently, many researchers have attempted to achieve EH with numerous harvesters and energy resources depending on the applications. While there are many sources for $\mathrm{EH}$, such as solar, wind, thermal, vibrational, temperature, and electromagnetic, RF energy is the most abundantly available, especially in urban environments. The massive adoption of RFEH can be owed to its ubiquitousness and reliability [1]. A basic block diagram representation of RF harvesting system is shown in Fig. 1.

A typical Rectenna consists of a transceiver antenna, optional Low pass filter, matching network, energy conversion unit and load/storage device. This sensitivity of antennas to $\mathrm{RF}$ signal induces an AC signal which is fed to the rectifier. The rectifier comprises diode(s) whose fast switching action is exploited to convert AC signal into DC. A low pass filter

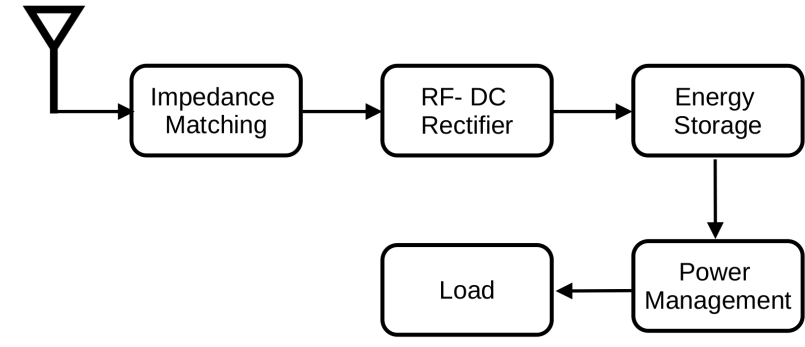

Fig. 1. Typical Block Digram Representation of RF Energy Harvesting System.

is employed for impedance matching between antenna and rectifier to achieve optimal power transfer. For an increased level of output voltages, a voltage multiplier can be employed. The storage and controlling unit provide an uninterrupted power supply. In contrast to other energy harvesters, RF harvesters are robust as they don't require any mechanical movements [2].

$\mathrm{RF}$ is an ambient source of energy, arising due to the radiations from TV broadcast, Radio (FM and AM), wireless LAN, Wireless Fidelity (Wi-Fi), and cellular transceiver stations [3]. Although ambient signals can be harvested with simple electronic circuitry, there are many challenges to be addressed by RF harvester:

- Since RF signals are available with wide frequencies, the RF harvester must ensure proper impedance matching for maximum power transfer.

- The RF should employ large broadband antennas to harvest valuable energy from the signals spread over a broad spectrum.

- The harvesting circuits must be positioned close to the RF power source since the ambient levels are deficient.

- The low energy density and low efficiency demand a dedicated RF energy supply as even a high-gain antenna cannot generate enough power densities.

With small-sized, high gain, and impedance-matched broadband antennas along with a reliable system for RF energy supply, energy harvesting in low power WSNs seem to be more promising and feasible.With the stupendous growth in mobile phones and Wi-Fi networks, RF energy has become pervasive and is significant in urban areas [4]. Wireless Power 
Transmission (WPT) can be classified into three categories, as described in Fig. 2.

The first category refers to near field inductive or resonant coupling. This reactive phenomenon occurs between two entities where the primary coil transfers power to the secondary. It is suitable for wireless charging of devices separated by few centimetres. The second category refers to far-field directive powering. Here power can be transmitted (in the form of RF) between two entities far from each other but has established a Line-of-Sight (LoS). For example, RF energy can be harvested from mobile phones in proximity, potentially providing poweron-demand for short-range sensing applications. This WPT is employed for the intentional powering of sensors equipped with a rectenna [5]. The third category refers to far-field $\mathrm{EH}$ where the receiver doesn't know where the RF energy is emitted (no LoS between the base station and the harvesting device). High gain antennas with wide beamwidth and wideband resonance are employed for enhanced and efficient energy harvesting in long-range operation.

The selection of the type of rectenna and the entire energy harvesting system varies from application to application [6], [7], [8]. Rectenna is a combination of rectifier and antenna. Diodes are used for rectification, while antenna can be either dipole, planar, or microstrip patch. Many attempts have been made to harvest energy from various RF signals. Among all the frequencies, $2.45 \mathrm{GHz}$ is the favourite. Most of our electronic devices love and live on this radio frequency, as it is in ISM bands (license is not required to operate in this band) [9]. It requires small antennas and can operate over long-range (with LoS). Our objective in this paper is double folded: first, review the attempts made in EH from RF signals and second, to design and develop a high-gain broadband antenna for harvesting over $2.45 \mathrm{GHz}$ signal.

The rest of the paper is ordered below: Section II provides a brief background with theoretical foundations and a literature review of rectennas employed in EH. Section III presents designs of 4-element micro-strip patch antennas for efficient EH. Section IV presents the discussion on designed antennas along with the merits and demerits of each design. Paper concludes in section V.

\section{BACKGROUND AND RELATED WORK}

Affordable and clean energy is the 7th SDG which aims to cater to the rising demands for energy while reducing the carbon footprint and burden on nature [10]. Energy harvesting seems to be one of the best prospects to realize this goal. EH refers to a process of capturing and storing the energy from sources around us that are free to use. $\mathrm{EH}$, also referred to as Energy Scavenging (ES), makes it possible to overcome the inconvenience of frequent replacement of batteries [11] while being less expensive and eco-friendly. $\mathrm{EH}$ stands as a viable solution for endless powering of low power loads such as wireless nodes.

Many attempts have been made to design EH schemes based on the availability of energy sources. Among all, RF$\mathrm{EH}$ is most suitable as the energy source is readily and abundantly available in transmitted energy. Other key benefits are being economically viable, eco-friendly, and having small form factor implementation [12]. RF-EH has the potential

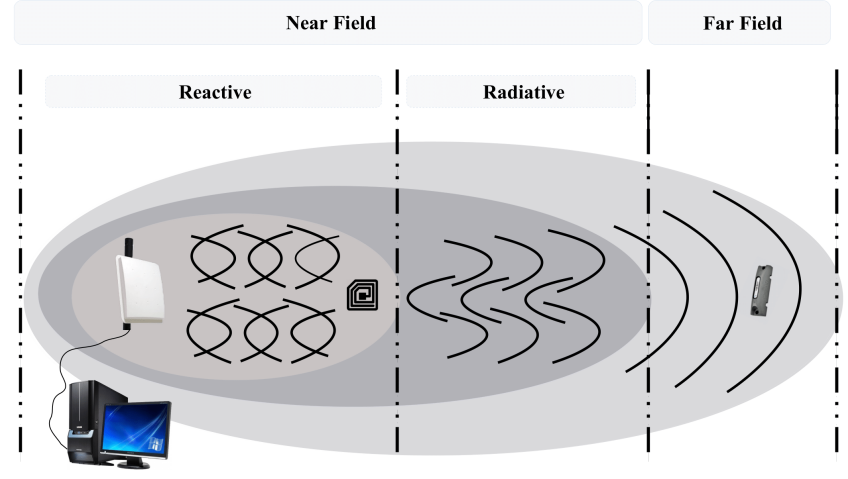

Fig. 2. Three Categories of WPT. Near Field, Far Field - Directional, and Far-Field Ambient Wireless Energy Harvesting

to revolutionize low-power applications - especially WSNs. Excessive use of batteries results in their disposal, causing extreme toxic pollution to the environment [13]. Furthermore, RF-EH can increase the lifetime of nodes and provide power indefinitely [14]. Passive energy scavenging nodes without batteries will be the next generation of WSNs, driven by RFEH because of its sustainability [15].

\section{A. Theoretical Foundations}

A proper understanding of EM waves is must while designing RF-EH system. EM waves largely vary w.r.t. distance, frequency, and conducting environment. Based on the application, designer need to take a call on the parameters of EM waves to make most out of the design. The relation between EM waves and distance from transmitting antenna can be categorized into two segments: near-field and far-field. These two fields are marked by Fraunhofer's distance given by

$$
d_{f}=\frac{2 D^{2}}{\lambda}
$$

where $d_{f}$ is fraunhofer distance, $\mathrm{D}$ is the maximum dimension of antenna, and $\lambda$ is wavelength of EM wave.

For a transceiver antenna, in far field the received power is given by

$$
P_{R}=\frac{P_{T} G_{T} G_{R} \lambda^{2}}{4 \pi R^{2}}
$$

where $P_{R}$ is power received; $G_{T}$ and $G_{R}$ are transmitter and receiver gains respectively.

The RF-DC conversion efficiency is given by

$$
\eta=\frac{V_{D C}^{2}}{P_{R} R_{L}} * 100
$$

where $V_{D C}$ is measured DC output voltage, $P_{R}$ is received $\mathrm{RF}$ input power and $R_{L}$ is resistive load. $P_{R}$ is given by $P_{D} *$ $A_{e f f}$ where $P_{D}$ is the RF power density and $A_{e f f}$ is effective aperture of antenna [16].

The RF power density for GSM900/1800 $\mathrm{MHz}$ is around $0.1 \mu \mathrm{W} / \mathrm{cm}^{2}$ while for $\mathrm{Wi}-\mathrm{Fi} 2.4 \mathrm{GHz}$ it is around $0.01 \mu \mathrm{W} / \mathrm{cm}^{2}$ Typically RF power conversion will be around $45 \%$ to $50 \%$ [17]. 


\section{B. Energy Harvesting Antenna Design}

Design of Energy harvesting antenna has attracted many researchers due to its sustainable and eco-friendly nature. A rectenna is a subsystem of wireless power transfer system which can be designed to function anywhere in the range of $1 \mathrm{GHz}$ to $35 \mathrm{GHz}$. Many factors such as transmitted power, transmitter gain, received power, receiver gain, conversion efficiency, will dictate the design of an energy harvesting antenna. To enhance the efficiency many other things, need to be considered and implemented such as arrays of antenna and circular polarization of antenna. The resonant frequency of a circular patch antenna is given by

$$
f_{r, n m}=\frac{\alpha_{n m} C}{2 \pi a_{e f f} \sqrt{\varepsilon_{r, e f f}}}
$$

where $\alpha_{n m}$ is the attenuation, $a_{e f f}$ is the effective relative dielectric constant.

Reconfigurable antennas got massive momentum recently due to their tuning, polarization, and selectivity of operating frequency. RF reconfigurability is achieved via dynamic modification of physical structure, thereby attaining polarization diversity. The advantage of automatic frequency tuning to accommodate wideband frequency makes reconfigurable antennas more popular. Though they seem promising, the other constraints like miniaturization, lightweight, beamforming, impedance matching, gain, radiation pattern need to be reworked every time the frequency is switched. While we finetune the performances of each module, the combination of all modules should be in harmony and result in efficient Wireless Power Harvesting (WPH) system.

Designing an efficient WPH system involves rigorous testing, making several adjustments, tuning many parameters, and evaluating the entire system. Operating frequency is the prime parameter that dictates the entire design. The operating range also needs to be specified. $\mathrm{GHz}$ frequencies are selected for long-range power harvesting, while $\mathrm{MHz}$ is sufficient for shortrange operations. In a dense environment, electromagnetic waves with very low frequency (in $\mathrm{kHz}$ ) is preferred. The topology of the electronic circuit(s) such as rectifier and voltage multiplier is decided based on the distance, operating frequency, and the required power output.

Antenna design is an essential part of the entire system design. In addition, rectifier and voltage multiplier design must match the power conversion efficiency. Though the capability of a WPH system critically depends on the antenna, it is one of the overlooked parts of energy harvesting system design. This slight inclination will significantly impact system performance as antennas are selected and deployed irrespective of the operating conditions, material to which it is attached and mobility of the tagged object.

To avoid degraded performance due to improper design of the antenna, the design process has to go through various steps as depicted in fig 3. Understanding the application and deploying environment will enable us to select operating frequency, bandwidth and required antenna parameters. These requirements determine the material for antenna construction

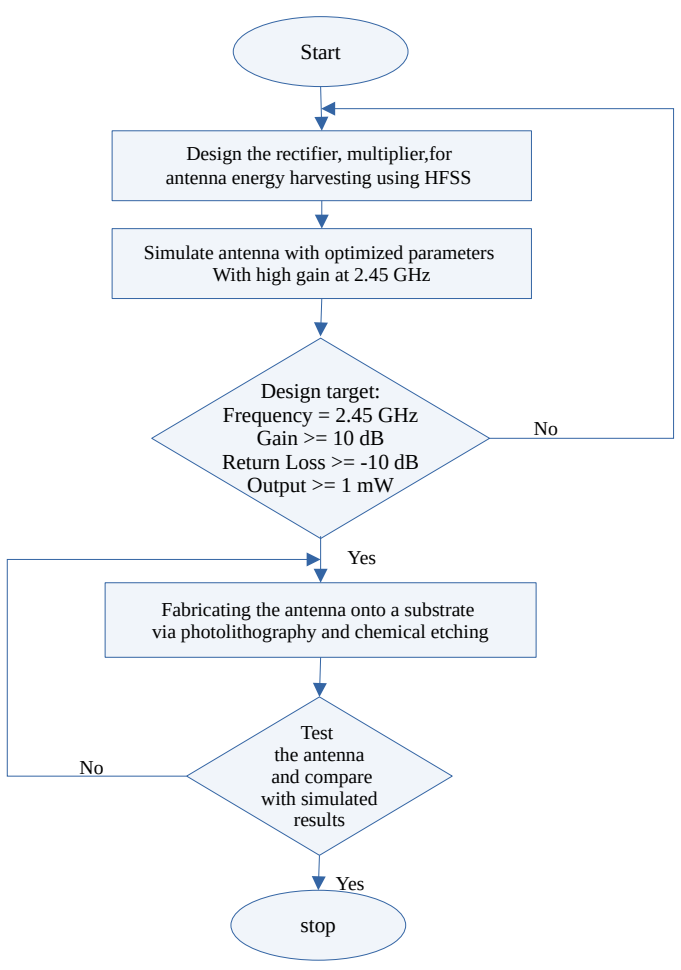

Fig. 3. RF Energy Harvesting Antenna Design Flow

and ASIC packaging. Antenna parametric study and optimization is done until design requirements are met in the simulation. The antenna is first modeled, simulated and optimized on a computer by monitoring the read range, antenna gain, and impedance which provides a good insight into antenna behavior. In the last step of the design process, prototypes are built, and their performance is measured extensively. If design requirement is satisfied, the antenna design is ready. Otherwise, the design is further modified and optimized until conditions are met.

\section{Previous Work}

Communication antennas have been explored for a century(since World War-I), However, energy harvesting antennas have got the momentum very recently. A narrowband antenna achieves very good energy conversion from RF to DC but can harvest only energy limited to few frequencies. On the other hand, wideband antennas retrieve a large amount of energy but come with large aperture size and poor conversion efficiency. One argument to multi-band antenna is at any given time an antenna cannot be made to resonate at two frequencies [18]. Table I summarizes the prior art of power-harvesting antennas.

Patch antennas have been explored extensively for harvesting energy from RF signal especially at $2.45 \mathrm{GHz}$ [19], [20], [22] and [25]. In [26], antennas with a resonant frequency of $2.45 \mathrm{GHz}$ and $5.8 \mathrm{GHz}$ were designed with Power Conversion Efficiencies (PCEs) of $65 \%$ and $46 \% @ 10 \mu \mathrm{W} / \mathrm{cm}^{2}$ Two different frequency bands i.e. 900/1800 MHz (for short range) GSM band and $2.4 \mathrm{GHz}$ ISM band were targeted by designing a microstrip antenna with joint feeding line implemented in a Multilayer substrate in [27]. Double patch 
TABle I. COMparison of Published Work Regarding Power-Harvesting Antennas

\begin{tabular}{|c|c|c|c|c|c|}
\hline Ref & Antenna Type & Freq. $(\mathrm{GHz})$ & Gain $(\mathrm{dBi})$ & Dimension $(\mathrm{mm})$ & RF-DC PCE \\
\hline [19] & Air-substrate Patch & 2.45 & 7 & $261 * 5$ & $30 \%$ \\
\hline$[20]$ & Patch & 2.45 & - & $100 * 70$ & $73.9 \% @ 207 \mu W / \mathrm{cm}^{2}$ \\
\hline [21] & Dual-Linearly Polarized Patch & 2.45 & $7.45-7.63$ & $70 * 47.5$ & $78 \% @ 295.3 \mu W / \mathrm{cm}^{2}$ \\
\hline [22] & Dual Polarized Patch & 2.45 & - & $100 * 100 * 3.8$ & $82.3 \% @ 22 \mathrm{dBm}$ \\
\hline [23] & Dipole & 2.45 & - & $60 * 60 * 60$ & $39 \% @ 0 \mathrm{dBm}$ \\
\hline [24] & Microstrip & 2.45 & 8.6 & - & $83 \%$ \\
\hline [25] & Patch & 2.45 & 4 & - & $70 \%$ \\
\hline [26] & Patch & 2.45 & 2.19 & $40 * 43$ & $65 \% @ 10 \mu W / \mathrm{cm}^{2}$ \\
\hline [27] & Microstrip & GSM band and ISM (2.45) & - & $72 * 94$ & $74 \% @ 0.3 \mu W / \mathrm{cm}^{2}$ \\
\hline [28] & Double Patch & 1.8 and 2.4 & - & $40 * 30$ & $19 \% @ 5 \mu W / \mathrm{cm}^{2}$ \\
\hline [29] & Single fed Microstrip patch & 2.4 & 7.19 & $60 * 60$ & $79 \%$ \\
\hline [30] & Microstrip patch Antenna Array $4 * 4$ & 35 & 19 & - & $67 \% @ 7 m W$ \\
\hline
\end{tabular}

${ }^{*}$ Both refers to inductive or capacitive

antenna was employed by [28] to operate at $1.8 \mathrm{GHz}$ and $2.4 \mathrm{GHz}$ with Simultaneous Wireless Information and Power Transfer (SWIPT) mechanism.

The arrangement of antennas in array is one of the best technique to achieve high gain and to obtain high voltage/current. Another advantage of array antennas over large aperture antennas is that they do not require large breakdown voltage diodes to operate. Connecting antenna array before rectification improves retrieved power at the main beam while placing the array after rectification will expands the ability to retrieve power from wide angles. Combining RF waves before rectification demands for a large breakdown diode, while combining RF waves after rectification, consolidating DC current will be an issue. Series connection of array antennas will enhance voltage whereas parallel fashion is opted for large current. Increasing the number of array elements will yield better outputs but reduces conversion efficiency.

\section{Microstrip Patch Antenna Design}

1) Estimation of Width of Patch Antenna: Width of microstrip antenna given by

$$
W_{p}=\frac{c}{2 f_{0} \sqrt{\frac{\epsilon_{r}+1}{2}}}
$$

Where $f_{o}$ is the operating frequency, $\mathrm{c}$ is the speed of light in air is $3 \times 10^{8} \mathrm{~m} \backslash \mathrm{sec}$ and $\epsilon_{r}$ is dielectric permitivity of substrate is 4.4

2) Estimation of Effective Dielectric Constant $\left(\epsilon_{\text {eft }}\right)$ : Where $\mathrm{h}$ is thickness or height of the substrate which is $1.6 \mathrm{~mm}$.

$$
\text { When } \frac{W_{p}}{h}>1 \text {, }
$$$$
\epsilon_{e f t}=\frac{\epsilon_{r}+1}{2}+\frac{\epsilon_{r}-1}{2}\left[\left(1+12 \frac{h}{W_{p}}\right)^{\frac{-1}{2}}+0.04\left(1-\frac{W_{p}}{h}\right)^{2}\right]
$$

When $\frac{W_{p}}{h}<1$,

$$
\epsilon_{e f t}=\frac{\epsilon_{r}+1}{2}+\frac{\epsilon_{r}-1}{2}\left[\left(1+12 \frac{h}{W_{p}}\right)^{\frac{-1}{2}}\right]
$$

3) Estimation of Effective Length $\left(L_{\text {eft }}\right)$ :

$$
L_{e f t}=\frac{c}{2 f_{o} \sqrt{\epsilon_{e f t}}}
$$

TABLE II. CALCULATED PARAMETERS FOR THE Microstrip PATCH ANTENNA

\begin{tabular}{ll}
\hline Parameters & Value \\
\hline Effective dielectric constant $\epsilon_{r}$ & 2.11 \\
Patch width $W$ & $49 \mathrm{~mm}$ \\
Patch length $L$ & $39.5 \mathrm{~mm}$ \\
Microstrip Line Length $y_{0}$ & $11.5 \mathrm{~mm}$ \\
Microstrip Line Width $W_{l}$ & $47 \mathrm{~mm}$ \\
Inset gap $W_{s}$ & $47 \mathrm{~mm}$ \\
Width of Substrate $W_{g}$ & $57 \mathrm{~mm}$ \\
Length of Substrate $W_{g}$ & $49 \mathrm{~mm}$ \\
\hline
\end{tabular}

4) Estimation of Length Extension $(\Delta L)$ :

$$
\Delta L=0.412 h \frac{\left(\epsilon_{e f t}+0.3\right)\left(\frac{W p}{h}+0.264\right)}{\left(\epsilon_{e f t}-0.258\right)\left(\frac{W p}{h}+0.8\right)}
$$
$\left(L_{p}\right)$ :

5) Estimation of Actual Length of Proposed Patch Antenna

$$
L_{p}=L_{e f t}-2 \Delta L
$$

6) Estimation of Ground Dimensions $\left(L_{g}, W_{g}\right)$ :

$$
L_{g}=6 h+L_{p}, W_{g}=6 h+W_{p}
$$

7) Estimation of Length of the Feed $\left(L_{f}\right)$ :

$$
L_{f}=\frac{\lambda_{g}}{4}, \lambda_{g}=\frac{\lambda}{\sqrt{\epsilon_{e f t}}}, \lambda=\frac{c}{f_{o}}
$$

Where $\lambda_{g}$ is Guide wavelength.

\section{Proposed Energy Harvesting Antenna}

The basic design of any patch antenna consists of three prominent parts. Ground, Substrate and the Patch. Though only patch works as a functional antenna, Ground and Substrate are added to provide physical support to the patch. In various cases Ground might be the base on which the antenna will be mounted and thus may be eliminated from the design. Substrate, unlike the ground is a must as it affects the radiation and bandwidth of the patch.

By using the above equations, we got the value of each dimension of the antenna, which is showed in Table II. Rectangular Patch antenna array with the dimensions mentioned in Table II is depicted in Fig. 4. 


\section{A. 4-Element Rectangular Patch Antenna Array}

The geometric parameters are adjusted to observe the variations with respect to the gain, bandwidth, and resonant frequency of the proposed antenna. The patch design incorporates several rectangular slots which are combined to form various shapes. The obtained frequencies is $2.4 \mathrm{GHz}$. The proposed antenna is designed by using the substrate RT/duroi 5880 substrate form Rogers. This substrate is characterized by 2.20 of dielectric constant with a dissipation factor of around 0.0004. Antenna array is designed using four patch elements with the aim of increasing the gain as shown in Fig. 4. Equidistant placement of the patch elements on the substrate forms a planar array. A feed network is used to connect patch elements and properly designed to enable equal radiation. Among all the available options one side feed network (all patch elements oriented in one direction) provides high gain, low loss, and single major beam with null deviation between electric and magnetic fields.

TABLE III. 4-ElEMENT H-Shaped Microstrip Patch ANTENNA ARRay Properties

\begin{tabular}{ll}
\hline Parameters & Value \\
\hline Path array dimensions & $119.5 * 118$ (in $\mathrm{mm})$ \\
Gain & $17.2 \mathrm{dBi}$ \\
Return Loss & -12.49 \\
Input impedance & $44+j 2.3$ \\
Bandwidth & $52 \mathrm{MHz}(\cong 2.1 \%)$ \\
\hline
\end{tabular}

\section{B. H-Shaped Patch Antenna Array}

Though the antenna is exhibiting acceptable behaviour, the bandwidth is not so superior as compared to previous works. In order to improve the bandwidth one need to tweak around the geometry of antennas without affecting other parameters and properties. Many techniques such as changing or removal (partial) of substrate, and introduction of slots either in radiating patch or ground plane have been investigated. In order to investigate the influence of various shapes and sizes of slots on bandwidth, simulations have been carried out. If we place a slot at the middle of the radiating edge, it may take the form of $\mathrm{U}$ or $\mathrm{H}$ shape as shown in fig 5 and 6 whose dimensions are mentioned in Tables III and IV. The simulation of $\mathrm{H}$ shape antenna array resulted in improved bandwidth.
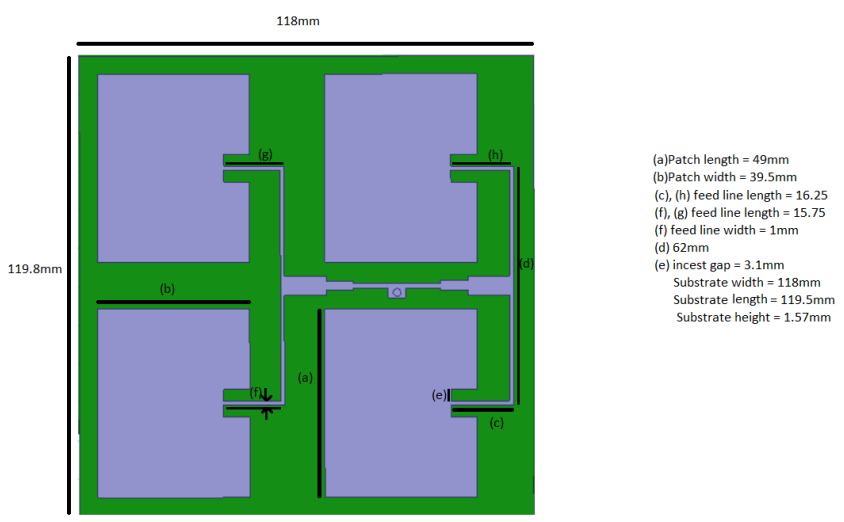

Fig. 4. 4-Element Rectangular Antenna Array

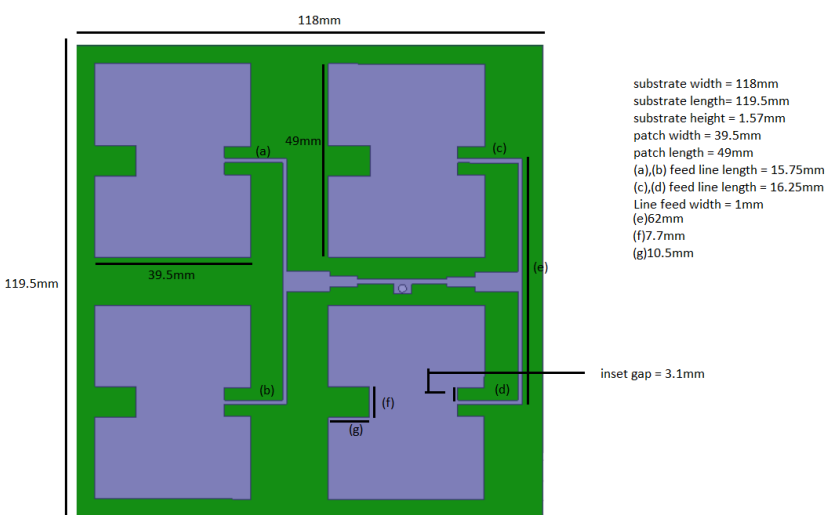

Fig. 5. 4-Element H-Shaped Patch Antenna Array

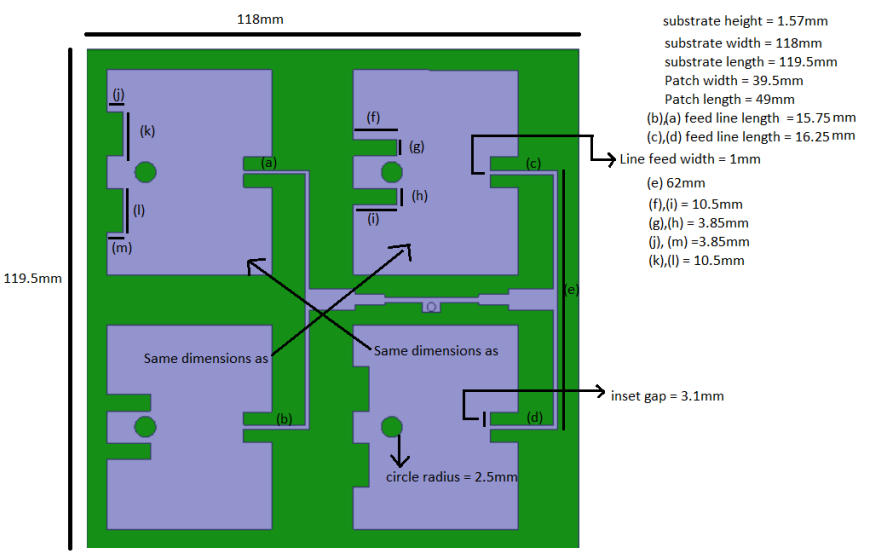

Fig. 6. 4-Element Modified H Shaped Patch Antenna Array

The width is designed as per the equations $W_{s}=\frac{\lambda}{60}$ and $L_{s}=\frac{c}{2 f \sqrt{\varepsilon_{e f f}}}-2\left(L+\Delta L-W_{s}\right)$.

TABLE IV. 4-ELEMENT MODIFIED H-SHAPED Microstrip Patch ANTENNA ARRAY Properties

\begin{tabular}{ll}
\hline Parameters & Value \\
\hline Path array dimensions & $119.5 * 118(\mathrm{in} \mathrm{mm})$ \\
Slot dimensions & $7.7 * 10.5(\mathrm{in} \mathrm{mm})$ \\
Gain & $17.2 \mathrm{dBi}$ \\
Return Loss & -12.49 \\
Input impedance & $40+j 5.5$ \\
Bandwidth & $64.8 \mathrm{MHz}(\cong 2.65 \%)$ \\
\hline
\end{tabular}

\section{Circular Slot, Modified H-Shaped Patch Antenna Array}

Though the focus will be on directivity and efficiency while designing an antenna (as shown in Fig. 7 and 8), in the larger picture, the aim will be to have great power reception and conversion (as shown in Fig. 9). Mutiband antennas are designed when energy has to be harvested from RF signals of wide range of frequencies (shown in Fig. 10). The authors major focus on enhancing bandwidth of the rectennas (4-element array) designed to harvest energy at a central frequency of $2.45 \mathrm{GHz}$. 


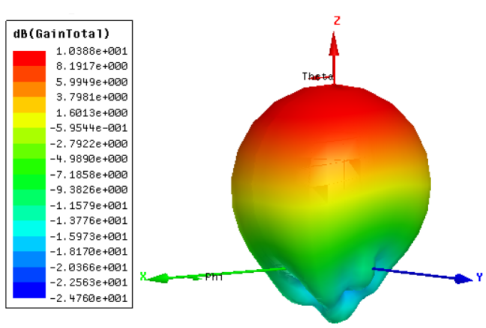

(a)

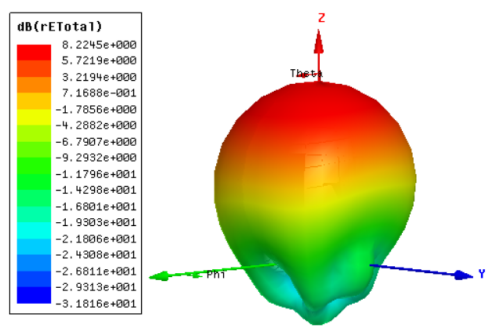

(b)

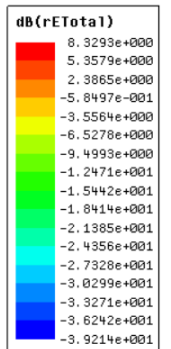

3. $924214 e+001$

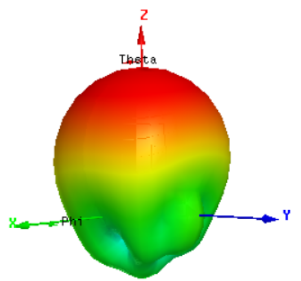

(c)

Fig. 7. 3D Polar Plots of (a) Single Microstrip Patch Element (b) H Shaped 4-Element Antenna Array (c) Modified H Shaped 4-Element Antenna Array

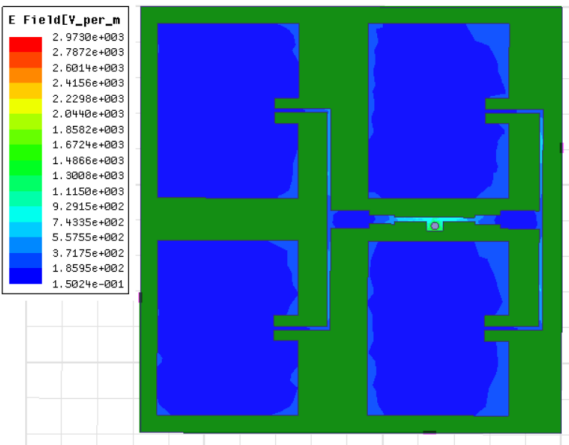

(a)

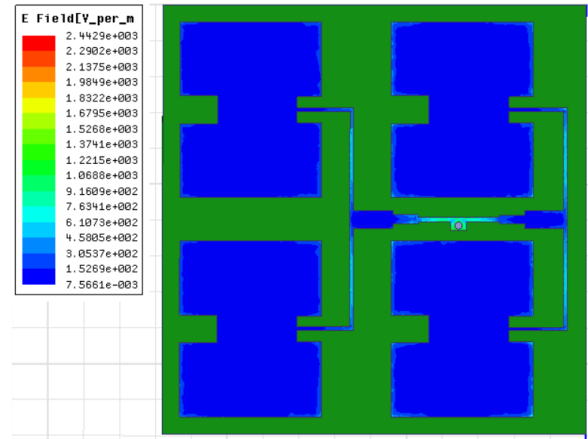

(b)
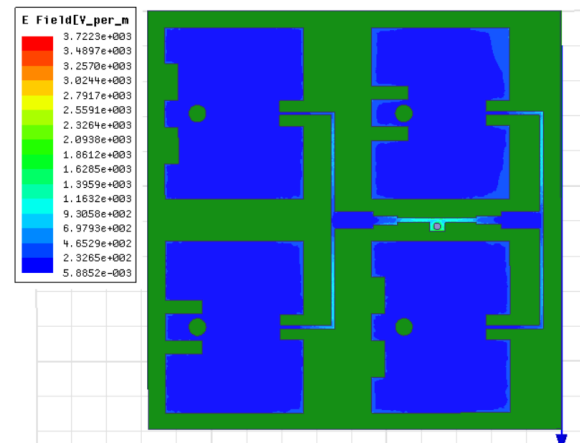

(c)

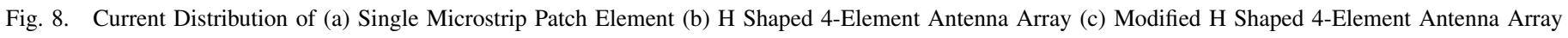

\section{RESUlts AND Discussion}

The IoT using wireless motes has perpetuated the demand for self-reliant electronics. Recent research has emphasized fulfilling this requirement via energy conservation. The energy crisis of these remotely placed devices needs to be taken care of. The energy crisis can be studied at various levels, either at energy resources level (choosing an appropriate and abundantly available energy resource), or at energy conservation level (energy-transformation mechanisms), or energy storage level (power management), or at energy consumption level (harvested energy is consumed responsibly).

Energy sources available for harvesting are thermal, solar, vibrational, chemical, Radio frequency, electromagnetic, and mechanical. All the sources demand bulkier devices with mechanical movements for harnessing the energy except RF. In contrast to all available sources, RF is ubiquitous, readily available, and is present in the ambience due to signal transmissions by all wireless transmitters. RF energy harvesting is much suited for IoT devices as there is huge restriction on size of both energy conversion devices and energy storage devices.

RF energy harvesting has garnered significant attention due to its consistent availability in the ambience from radio, TV, Cellular and Wi-Fi communications. Many energy-saving mechanisms are being investigated, including Radio optimization, Data optimization, scheduling schemes, Routing and Topology Control, and messaging protocols. Among all options, Radio optimization has shown huge potential in energy saving as it deals with energy harvesting. Radio optimization tries to save energy via transmission power control, Directional antenna, and Cognitive Radio.
Wireless transmission of energy has no bounds. Wireless power transfer is the transmission of electrical energy from a transmitter connected to a power source via beamforming to one or more receivers without power cords. At the receiver, the electromagnetic signal is converted back to an electric current and then used by either 1) inductive, capacitive or resonant reactive near-field coupling, or 2) far-field directive power beamforming, or 3) far-field non-directive power transfer.

Since near field and far field with line-of-sign are quiet conducive for energy harvesting, it is implied that much of the research should be focused on the third type i.e. far-field nonLoS WPT. The two challenges in such deployment is the low power densities of incident power and the dynamics of position and orientation of the reciever. The sudden variations in the location brings in much chaos in the power levels which can be addressed by a designing a recitifier capable of operating over a wide-range of incident power. The challenge of low power densities can be partially overcome by having high RF-DC power conversion efficiency (PCE). But it is should be noted that, if more energy is sucked or scavenged from the ambience, the RF-DC will result in much higher PCE. Hence, Rectennas (reciever antennas with rectifiers) need to be designed with broad band to scavenge large amount of low power energy from the ambience such that the voltage multiplier will boost the level and rectifier will take care of the variation in levels.

The learning from various sections of this work can be summarized as below:

1) Energy concerns in IoT devices can be rightly addressed by employing energy harvesting mechanisms.

2) Among all available sources of energy, RF energy 


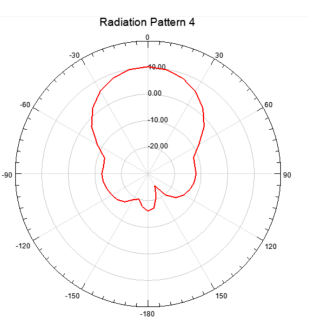

(a)

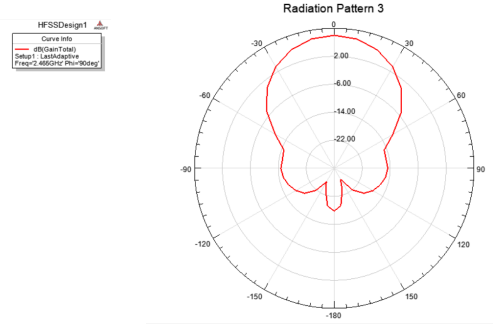

(b)

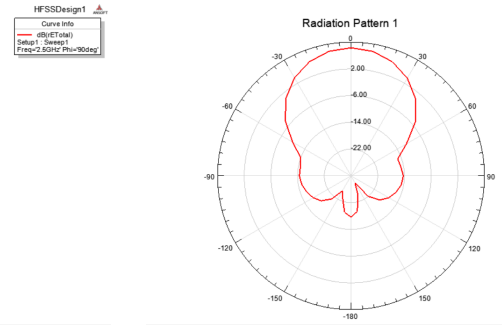

(c)

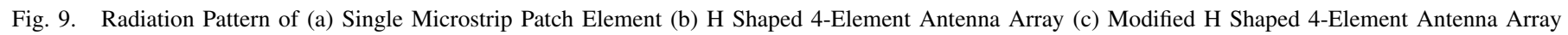

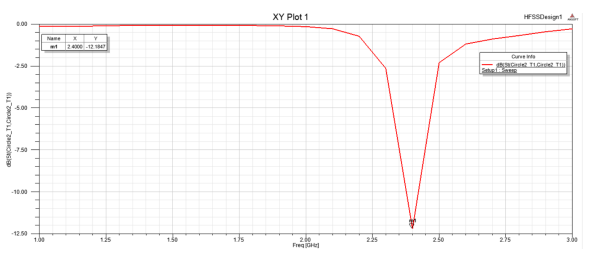

(a)

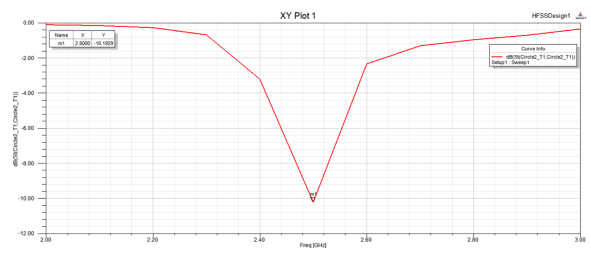

(b)

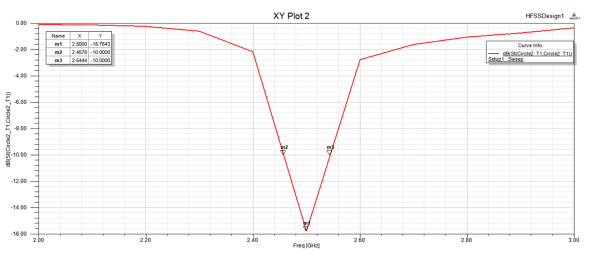

(c)

Fig. 10. Return Loss of (a) Single Microstrip Patch Element (b) H Shaped 4-Element Antenna Array (c) Modified H Shaped 4-Element Antenna Array

table V. 4-Element Patch Antenna Array Properties

\begin{tabular}{llll}
\hline Parameters & $\begin{array}{l}\text { Value of Rectangular Patch } \\
\text { antenna array }\end{array}$ & $\begin{array}{l}\text { Value of H-shaped Patch } \\
\text { antenna array }\end{array}$ & $\begin{array}{l}\text { Modified H-shaped antenna } \\
\text { with circular slot }\end{array}$ \\
\hline Path array dimensions & $119.5 * 118$ (in $\mathrm{mm})$ & $119.5 * 118$ (in mm) & $119.5 * 118$ (in mm) \\
Slot dimensions & Not applicable & $7.7 * 10.5$ (in mm) & $7.7 * 10.5$ (in mm) \\
Gain & $10.4 \mathrm{dBi}$ & $8.225 \mathrm{dBi}$ & $8.33 \mathrm{dBi}$ \\
Return Loss & -12.49 & -10.49 & -15.49 \\
Bandwidth & $258 \mathrm{MHz}(\cong 10.75 \%)$ & $64.8 \mathrm{MHz}(\cong 2.65 \%)$ & $868 \mathrm{MHz}(\cong 36.17 \%)$ \\
\hline
\end{tabular}

harvesting is best due to its ubiquitousness, and simple design requirement without any mechanical movements and no demand for large storage.

3) Among all available ambient frequencies, $2.4 \mathrm{GHz}$ is best for long-range wireless power transmission.

4) For a central operating frequency of $2.4 \mathrm{GHz}, \mathrm{Mi}-$ crostrip patch antenna is best suited.

5) One side feed network is provides better results.

6) 4-element antenna array is best arrangement for energy harvesting in low-power applications.

7) Creating a circular slot is best option for increasing the bandwidth instead of going for multi-band antennas (which have their own limitation of switching) as demonstrated in this work.

This work has attempted to demonstrate the development of receiving antenna array design starting from a basic rectangular patch antenna. A simple rectangular patch antenna is designed with 4 elements to form an array. The parameters are optimized by the equations defined in Section II, taken from [16]. The centre frequency of $2.4 \mathrm{GHz}$ is accurately achieved. Motivated by this, the authors have attempted to recreate a $\mathrm{H}$-shaped antenna with small improvement in bandwidth from the work [31]. Here a small $\mathrm{H}$ shaped antenna, based on tuned slot size is designed. The simulation result shows an incremental change in bandwidth i.e. from $2.1 \%$ to $2.65 \%$. Even if we appreciate the delta enhancement, the practical results were not so encouraging as demonstrated in [31]. Therefore inorder to have large bandwidth, the authors have introduced a circular slot in the patch antenna. This inclusion of circular slot has shown remarkable change in bandwidth i.e. from 52.2
$\mathrm{MHz}$ (for rectangular patch antenna array) and $64.8 \mathrm{MHz}$ (for $\mathrm{H}$-shaped patch antenna array) to a large bandwidth of 868 $\mathrm{MHz}$. This accounts to $36.17 \%$ bandwidth against the $2.65 \%$ of H-shaped antenna. This new antenna array can be explored for intelligent beamforming [32]. The comparison of results is tabulated in Table V.

\section{CONClusion}

Given the SDGs, energy crisis is inevitable with the way resources are being exploited. In a low power device placed remotely, energy scavenging is the preferred mechanism to enhance the lifetime of the node. This work considers RF signals to harvest at $2.4 \mathrm{GHz}$, which is readily available and free to use. The antenna design at this frequency is selected to be microstrip patch with a suitable one side feed network. The work has considered bandwidth to enhance the power conversion efficiency by designing a wideband rectenna with 4-element arrangement for energy harvesting in lowpower devices such as IoT devices, Radio Frequency Identifier (RFID) and remote wireless motes.

Energy concerns in IoT devices can be rightly addressed by employing energy harvesting mechanisms. Among all available sources of energy, RF energy harvesting is best due to its ubiquitousness, and simple design requirement without any mechanical movements and no demand for large storage. In all the available ambient frequencies, $2.4 \mathrm{GHz}$ is best for longrange wireless power transmission. For a central operating frequency of $2.4 \mathrm{GHz}$, Microstrip patch antenna is best suited. One side feed network provides better results. 4-element antenna array is best arrangement for energy harvesting in 
low-power applications. Creating a circular slot is best option for increasing the bandwidth instead of going for multi-band antennas (which have their own limitation of switching) as demonstrated in this work. The simple flow from rectangular antenna to the circularly slotted modified H-shape antenna along with the theoretical foundations and the antenna design flow chart, this work acts as a primer for any communication engineer enthusiast to start simulating various slots and enhance various properties of antennas without affecting the other parameters. The authors are confident that the fabricated antenna would give better results and may provide a bandwidth enhancement of at least 20\% while considering all nonlinearities and implementation losses.

This work has paved a way towards radio optimization and can be extended to transmitter side, where beamforming for energy transmission with receiver location aware precoding can be explored. The authors are confident that the fabricated antenna would give better results and may provide a bandwidth enhancement of at least $20 \%$ while considering all non-linearities and implementation losses. The authors also look forward to work on other modules of rectenna: Rectifier, voltage multiplier, power divider, and power management schemes in Wireless Sensor Networks.

\section{ACKNOWLEDGMENT}

The authors would like to thank the anonymous reviewers of this work, and the Doctoral Committee members of VTU, Belagavi, whose suggestions and insights have shaped this work.

\section{REFERENCES}

[1] Adu-Manu, Kofi Sarpong, et al. "Energy-harvesting wireless sensor networks (EH-WSNs) A review." ACM Transactions on Sensor Networks (TOSN) 14.2 (2018): 1-50.

[2] Zeadally, Sherali, et al. "Design architectures for energy harvesting in the Internet of Things." Renewable and Sustainable Energy Reviews 128 (2020): 109901.

[3] Sangare, Fahira, and Zhu Han. "RF Energy Harvesting Networks: Existing Techniques and Hardware Technology." Wireless Information and Power Transfer: A New Paradigm for Green Communications. Springer, Cham, 2018. 189-239.

[4] Khemar, Adel, et al. "Design and experiments of a dual-band rectenna for ambient RF energy harvesting in urban environments." IET Microwaves, Antennas \& Propagation 12.1 (2018): 49-55.

[5] Costanzo, Alessandra, and Diego Masotti. "Wirelessly powering: An enabling technology for zero-power sensors, IoT and D2D communication." 2015 IEEE MTT-S International Microwave Symposium. IEEE, 2015.

[6] Sanil, Nischal, Pasumarthy Ankith Naga Venkat, and Mohammed Riyaz Ahmed. "Design and performance analysis of multiband microstrip antennas for IoT applications via satellite communication." 2018 Second International Conference on Green Computing and Internet of Things (ICGCIoT). IEEE, 2018.

[7] Punith, S., et al. "A Novel Multiband Microstrip Patch Antenna for 5G Communications." Procedia Computer Science 171 (2020): 2080-2086.

[8] Sanil, Nischal, Pasumarthy Ankith Naga Venkat, and Mohammed Riyaz Ahmed. "Design of an U shaped slotted patch antenna for RFID Vehicle Identification.” 2018 Second International Conference on Green Computing and Internet of Things (ICGCIoT). IEEE, 2018.

[9] Firestone, Mary. Wireless technology. Lerner Publications, 2008.

[10] Assembly, General. "Sustainable development goals." SDGs Transform Our World 2030 (2015).

[11] Anjum, Shaik Shabana, et al. "Energy management in RFID-sensor networks: Taxonomy and challenges." IEEE Internet of Things Journal 6.1 (2017): 250-266.
[12] Williams, Alexander J., et al. "Survey of Energy Harvesting Technologies for Wireless Sensor Networks." IEEE Access (2021).

[13] Dehghani-Sanij, A. R., et al. "Study of energy storage systems and environmental challenges of batteries." Renewable and Sustainable Energy Reviews 104 (2019): 192-208.

[14] Ahmed, Sheeraz, et al. "Energy harvesting techniques for routing issues in wireless sensor networks." International Journal of Grid and Utility Computing 10.1 (2019): 10-21.

[15] Perera, Tharindu D. Ponnimbaduge, et al. "Simultaneous wireless information and power transfer (SWIPT): Recent advances and future challenges." IEEE Communications Surveys \& Tutorials 20.1 (2017): 264-302.

[16] Balanis, Constantine A. Antenna theory: analysis and design. John wiley \& sons, 2015

[17] Piñuela, Manuel, Paul D. Mitcheson, and Stepan Lucyszyn. "Ambient RF energy harvesting in urban and semi-urban environments." IEEE Transactions on microwave theory and techniques 61.7 (2013): 27152726.

[18] Kandakatla, Radha Anil, and Mohammed Riyaz Ahmed. "Design and Performance Analysis of Dual-band Microstrip patch antennas for Smart Apparels.” 2018 Second International Conference on Green Computing and Internet of Things (ICGCIoT). IEEE, 2018.

[19] Momenroodaki, Parisa, Ricardo Dias Fernandes, and Zoya Popović. "Air-substrate compact high gain rectennas for low RF power harvesting." 2016 10th European conference on antennas and propagation (EuCAP). IEEE, 2016.

[20] Sun, Hucheng. "An enhanced rectenna using differentially-fed rectifier for wireless power transmission." IEEE Antennas and Wireless Propagation Letters 15 (2015): 32-35.

[21] Sun, Hucheng, and Wen Geyi. "A new rectenna with all-polarizationreceiving capability for wireless power transmission." IEEE Antennas and Wireless Propagation Letters 15 (2015): 814-817.

[22] Chou, Jui-Hung, et al. "All polarization receiving rectenna with harmonic rejection property for wireless power transmission." IEEE Transactions on Antennas and Propagation 62.10 (2014): 5242-5249.

[23] Niotaki, Kyriaki, et al. "A compact dual-band rectenna using slotloaded dual band folded dipole antenna." IEEE Antennas and Wireless Propagation Letters 12 (2013): 1634-1637.

[24] Sun, Hucheng, et al. "Design of a high-efficiency $2.45-\mathrm{GHz}$ rectenna for low-input-power energy harvesting." IEEE Antennas and Wireless Propagation Letters 11 (2012): 929-932.

[25] Olgun, Ugur, Chi-Chih Chen, and John L. Volakis. "Wireless power harvesting with planar rectennas for $2.45 \mathrm{GHz}$ RFIDs." 2010 URSI International Symposium on Electromagnetic Theory. IEEE, 2010.

[26] Ren, Yu-Jiun, Muhammad F. Farooqui, and Kai Chang. "A compact dual-frequency rectifying antenna with high-orders harmonic-rejection." IEEE Transactions on Antennas and Propagation 55.7 (2007): 2110-2113.

[27] Shuvo, Md Abdul Kader, and Md Mahmudul Hasan. "Multi-Band Microstrip Antenna Design for Wireless Energy Harvesting." American Journal of Energy and Environment 3.1 (2018).

[28] Silva, Vinícius S., et al. "Double Patch Antenna Array for Communication and Out-of-band RF Energy Harvesting." Journal of Microwaves, Optoelectronics and Electromagnetic Applications 19 (2020): 356-365.

[29] Kumar, N. Rajesh, and P. D. Sathya. "Design of RF Energy Harvesting Patch Antenna for Wireless Communications."

[30] Mavaddat, Ali, Seyyed Hossein Mohseni Armaki, and Ali Reza Erfanian. "Millimeter-Wave Energy Harvesting Using $4 \times 4$ Microstrip Patch Antenna Array." IEEE Antennas and wireless propagation letters 14 (2014): 515-518

[31] Chaour, Issam, Ahmed Fakhfakh, and Olfa Kanoun. "Patch Antenna Array for RF Energy Harvesting Systems in $2.4 \mathrm{GHz}$ WLAN Frequency Band." 2018 15th International Multi-Conference on Systems, Signals \& Devices (SSD). IEEE, 2018.

[32] Nalband, Abdul Haq, Mrinal Sarvagya, and Mohammed Riyaz Ahmed "Optimal hybrid precoding for millimeter wave massive MIMO systems." Procedia Computer Science 171 (2020): 810-819. 